\title{
La fondation de la philologie du provençal, une affaire franco-allemande
}

\author{
Jacques FRANÇOIS \\ Université de Caen, LaTTiCe UMR 8094 \\ jfrancois@interlingua.fr / www.interlingua.fr
}

\begin{abstract}
Résumé. Après les premières observations au $18^{\mathrm{e}}$ siècle de La Curne de Saint-Palaye sur l'ancien provençal et sa littérature, l'étude philologique et linguistique de la «langue des troubadours »a intéressé tout au long du $19^{\mathrm{e}}$ siècle des savants français et allemands au fil d'un dialogue renouvelé d'une génération à l'autre. Les principaux protagonistes de la première génération ( $\mathrm{F}$. Raynouard, A.W. Schlegel, F. Diez) ont déblayé un terrain philologique particulièrement riche et jeté les bases de la grammaire de cette langue, Raynouard allant jusqu'à lui attribuer le statut de langue 'romane', conçue comme un passage obligé entre le 'latin rustique' et les langues romanes en émergence. Les savants de la seconde génération (G. Paris et P. Meyer côté français, K. Bartsch, K.A.F. Mahn, E. Levy, H. Suchier côté allemand) se sont donné pour tâche d'aller au-delà du monumental Lexique roman de Raynouard (à partir de 1838). Au tournant du $20^{\mathrm{e}}$ siècle, les chercheurs, encouragés par la renommée croissante du Félibrige, disposaient d'un outillage grammatical et lexicographique exceptionnel, fruit de cette collaboration francoallemande, mais l'ancien provençal avait perdu son charme de belle langue au bois dormant.
\end{abstract}

\begin{abstract}
This paper aims at assessing the respective contribution of German and French romanists to the study of Provençal as the "language of troubadours". The story begins at the end of $18^{\text {th }}$ century with La Curne de Saint-Palaye, goes on with the collaborative work of F. Raynouard, A.W. Schlegel and F. Diez in the first half of $19^{\text {th }}$ century and the competition comes to an end with the second generation scholars, G. Paris and P. Meyer on the French side, as well as K. Bartsch, K.A.F. Mahn, E. Levy and H. Suchier on the German side. This story ilustrates the passionate interest of German linguists in the genesis of the Romance language family till now.
\end{abstract}

\section{Les protagonistes des deux côtés du Rhin}

« Raynouard apparut et la lumière se fit ». Ainsi s'exprime Ferdinand de RoISIN dans sa préface à l'Essai sur les cours d'amour de Friedrich DIEZ (cf. Diez 1845, p.xij) à propos de la redécouverte de l'œuvre poétique des troubadours, après avoir évoqué les prémisses de cette entreprise philologique, notamment chez Jean-Baptiste de LA CURNE DE SAINT-PALAYE (1697-1781). Plus explicitement, Roisin évoque (p.xj) la filiation entre le Choix des poésies originales des troubadours de François Juste-Marie RAYNOUARD (tome 1,1816) et l'Essai de Diez :

C'est au banc des demandeurs, la révision consciencieuse des débats, l'exposé rationnel de nouveaux moyens, c'est en un mot une seconde exploration dans le territoire reconnu par Raynouard. Le digne émule du grand maître vérifie les jalons d'attente, affermit les uns, transplante les autres, en pose à son tour, et agrandit ainsi le domaine acquis. 
Cependant l'ouvrage original de Diez date de 1825 et est donc postérieur de sept ans à celui d'August Wilhelm SCHLEGEL (1767-1845) rédigé en français, Observations sur la langue et la littérature provençales (1818). Concernant la philologie provençale (ou occitane), A.W. Schlegel est donc un maillon intermédiaire entre le précurseur, La Curne, le pionnier, Raynouard (1761-1836), et la figure de proue, Diez.

Dès le second quart du $19^{\mathrm{e}}$ siècle, les principaux spécialistes de la philologie provençale sont des héritiers de Diez, essentiellement en Allemagne, notamment Karl August Friedrich MAHN (1802-1887), Karl BARTSCH (1832-1888) et Emil LEVY (1855-1917), mais aussi en France, où Gaston PARIS (1839-1903) se fait le porte-voix efficace ${ }^{1}$ de l'œuvre de Diez en traduisant l'Introduction (1863) puis la totalité (18741876) de sa Grammaire des langues romanes. Le tableau 1 propose un découpage en cinq phases de l'histoire de la compétition entre philologues français et allemands dans l'étude littéraire, mais préalablement lexicale et grammaticale, de la «langue des troubadours » que Raynouard tenait pour un maillon essentiel entre le latin 'rustique' et les langues romanes en émergence.

\begin{tabular}{|c|c|c|c|}
\hline & PHILOLOGUES FRANÇAIS & DATE & PHILOLOGUES ALLEMANDS \\
\hline 1 & $\begin{array}{l}\text { Jean-Baptiste de LA CURNE DE SAINT-PALAYE, } \\
\text { Histoire littéraire des troubadours } \\
\end{array}$ & 1774 & \\
\hline \multirow{7}{*}{2} & $\begin{array}{c}\text { François RAYNOUARD, Choix des poésies } \\
\text { originales des troubadours, vol.1 }{ }^{2}\end{array}$ & 1816 & \\
\hline & & 1818 & $\begin{array}{l}\text { August-Wilhelm SCHLEGEL, Observations } \\
\text { sur la langue et la littérature provençales }\end{array}$ \\
\hline & $\begin{array}{l}\text { Henri Pascal de ROCHEGUDE, Essai d'un glossaire } \\
\text { occitanien : pour servir à l'intelligence des } \\
\text { poésies des troubadours }\end{array}$ & 1819 & \\
\hline & & 1820 & $\begin{array}{l}\text { Friedrich DIEZ, Compte rendu de Raynouard } \\
1816 \text { et Schlegel } 1818 \text { (éd. 1882) }\end{array}$ \\
\hline & \multirow[t]{3}{*}{$\begin{array}{l}\text { François RAYNOUARD, Grammaire comparée des } \\
\text { langues de l'Europe latine, dans leurs rapports } \\
\text { avec la langue des troubadours }\end{array}$} & 1821 & \\
\hline & & 1825 & $\begin{array}{l}\text { Friedrich DIEZ, Über die Minnehöfe, } \\
\text { Beiträge zur Kenntnis der romanischen } \\
\text { Poesie; (fr.1842) Essai sur les cours } \\
\text { d'amour }\end{array}$ \\
\hline & & 1829 & $\begin{array}{l}\text { Friedrich DIEZ, Leben und Werke der } \\
\text { Troubadours }\end{array}$ \\
\hline \multirow[b]{2}{*}{3} & $\begin{array}{l}\text { François RAYNOUARD, Influence de la langue } \\
\text { romane rustique sur les langues de l'Europe latine }\end{array}$ & 1836 & $\begin{array}{l}\text { Friedrich DIEZ, Grammatik der } \\
\quad \text { romanischen Sprachen (3 tomes 1836-38) }\end{array}$ \\
\hline & $\begin{array}{l}\text { François RAYNOUARD, Lexique roman ou } \\
\text { Dictionnaire de la langues des troubadours } \\
\text { comparée avec les autres langues de l'Europe } \\
\text { latine, tome } 1\end{array}$ & 1838 & $\begin{array}{l}\text { (cf. Tome } 2 \text { pp.187-207; tome 3, pp.283-339) } \\
\text { Trad. fr. par G. Paris et A. Brachet (1874- } \\
\text { 1876). }\end{array}$ \\
\hline
\end{tabular}

Tableau 1a : La contribution des philologues français et allemands à l'étude de l'ancien provençal (jusqu'en 1838) 


\begin{tabular}{|c|c|c|c|}
\hline \multirow{4}{*}{4} & & 1854 & $\begin{array}{l}\text { Karl BARTSCH, Provenzalisches Lesebuch, mit einer } \\
\text { literarischen Finleitung und einem Wörterbuche }\end{array}$ \\
\hline & \multirow{3}{*}{$\begin{array}{l}\text { Gaston PARIS, trad. de l'Introduction à la } \\
\text { Grammaires des langues romanes de Fr. } \\
\text { Diez } \\
\text { Gaston PARIS, La philologie romane en } \\
\text { Allemagne [Bibl. de l'École des Chartes] \& } \\
\text { Introduction à la Grammaires des langues } \\
\text { romanes de Fr. Diez }\end{array}$} & 1863 & $\begin{array}{l}\text { Karl August Friedrich MAHN, „Ueber die Entstehung, } \\
\text { Bedeutung, Zwecke und Ziele der romanischen } \\
\text { Philologie“ [cité par Paris 1864] }\end{array}$ \\
\hline & & 1864 & \\
\hline & & 1868 & Karl BARTSCH, Chrestomathie provençale \\
\hline \multirow{4}{*}{5} & & 1885 & $\begin{array}{l}\text { Karl August Friedrich MAHN, Lautlehre der } \\
\text { altprovençalischen Sprache }\end{array}$ \\
\hline & & 1894 & $\begin{array}{l}\text { Emil LEVY, Provenzalisches Supplement-Wörterbuch } \\
\text { - Berichtigungen und Ergänzungen zu Raynouards } \\
\text { Lexique Roman }\end{array}$ \\
\hline & & 1904 & $\begin{array}{l}\text { Hermann SUCHIER, Die französische und } \\
\text { provenzalische Sprache und ihre Mundarten (p.712- } \\
\text { 841) [in Gustav GRÖBER, ed. Grundriss der } \\
\text { romanischen Philologie, vol.1, 2e éd.] }\end{array}$ \\
\hline & & 1909 & Emil LEVY, Petit dictionnaire provencal-francais \\
\hline
\end{tabular}

Tableau 1b : La contribution des philologues français et allemands à l'étude de l'ancien provençal (à partir de 1854)

Le tableau 1a-1b se subdivise en cinq époques :

- La première est constituée par l'édition ${ }^{3}$ posthume en 1774 par l'Abbé Millot de papiers de J.B. de La Curne de Saint-Palaye consacrés à l'histoire littéraire des troubadours. Dans les quatre époques suivantes, les travaux des philologues français et allemands se sont concurrencés avec un succès variable.

- La seconde (1816-1829) est celle des premiers travaux croisés de F. Raynouard (1816, 1821), d'A.W. Schlegel (1818) recensant Raynouard (1816), de F. Diez recensant à son tour Raynouard (1816) et Schlegel (1818) avant de disserter sur les cours d'amour (1825, ouvrage traduit en français en 1842 avec une intéressante préface de son traducteur F. de Roisin) et la vie et les œuvres des troubadours (1829), auxquels il faut ajouter le Glossaire occitanien de H.P. de Rochegude (1819). Dans cette première période, Diez se concentre sur la prouesse littéraire des troubadours, estimant manifestement que Raynouard en a suffisamment traité les bases grammaticales et lexicales.

- La troisième, très brève, période (1836-1838) est la plus intéressante pour notre sujet : elle concerne d'un côté Raynouard qui publie en 1836 un opuscule sur l'influence de la « langue romane rustique » sur les langues de l'Europe latine et en 1838 le premier volume de son Lexique roman, et de l'autre Diez qui publie dans le même temps son œuvre majeure, la Grammatik der romanischen Sprachen en trois volumes, avec des observations détaillées sur la morphologie du provençal dans le volume 2.

- Dans la quatrième période (1854-1868), de nouveaux protagonistes apparaissent, essentiellement du côté allemand avec Karl Bartsch, auteur en 1854 d'une anthologie de la poésie provençale pour le public allemand et en 1868 d'une Chrestomathie provençale pour le public français avec un abrégé grammatical (p.413-433) et surtout un impressionnant glossaire de quelque 6000 mots (p.438-574), et K.A.F. Mahn, auteur entre autres en 1863 d'une conférence mémorable sur la naissance, la signification et les objectifs de la philologie romane ; mais aussi du côté français avec G. Paris qui traduit en 1863 l'introduction de la Grammaire des langues romanes de Diez (avant d'en publier avec A. Brachet la traduction complète en trois volumes en 1874-1876) et qui, dans un article paru en 1864 
dans les Annales de la bibliothèque de l'École des Chartes, rend hommage à la conférence de Mahn et encourage ses collègues archivistes-paléographes à suivre la voie ouverte par Diez et Mahn.

- Enfin la cinquième période (1885-1909) est caractérisée par le désintérêt des philologues français pour la linguistique du provençal, question qu'ils laissent les Allemands creuser à leur place, avec la phonétique de l'ancienne langue provençale de K.A.F. Mahn en 1885 et les deux dictionnaires du provençal d'A. Levy, d'abord en allemand comme supplément au Lexique roman de Raynouard (1894), puis en français (1909) avec le Petit dictionnaire provençal-français. Dans le même temps cependant des indices (cf.\$5) suggèrent que l'époque de l'investigation systématique de la langue provençale est arrivée à son terme.

\section{Le début du dialogue entre romanistes français et allemands sur l'ancien provençal (1816-1829)}

\section{- RAYNOUARD (1816)}

Comme le détaille le sous-titre du premier volume du Choix des poésies originales des troubadours de François Raynouard, ce premier ouvrage se compose d'une introduction de 32 pages et de trois sections portant respectivement sur [1] les preuves historiques de l'ancienneté de la langue romane (pp.1-32), [2] les recherches sur l'origine et la formation de cette langue et les éléments de sa grammaire avant l'an 1000 (pp.33-108) et [3] la grammaire de la langue des troubadours (pp.109-437), suivies d'un appendice (p.438-447) bibliographique et grapho-phonétique.

Concernant l'origine de la «langue romane », Raynouard défend une thèse en deux points : [1] le latin 'rustique' [futur latin 'vulgaire'] s'est répandu à travers toute la partie de l'empire carolingien où il n'était pas en concurrence avec le 'théotisque' (ou francique). Raynouard en donne de nombreux indices pour l'Espagne, la Gaule et l'Italie; [2] La France méridionale (l'Aquitaine au sens large) est l'aire linguistique où le latin rustique a été épuré en premier, d'où l'émergence de la littérature provençale. Dans l'introduction, il propose trois méthodes de mise en évidence de cette «langue romane » (p.xxiii sq):

Soit en recherchant les traces les plus reculées de l'emploi de l'article qui a été l'un des caractères innovateurs de cet idiôme ; soit en reconnaissant les noms propres qui, dans les ouvrages latins écrits à une époque ancienne, sont désignés par une dénomination purement romane ; soit enfin en cherchant dans les écrits de la basse latinité, les traces de la réaction de la langue vulgaire sur la langue latine.

Dans la section consacrée à l'origine et à la formation de la langue romane, Raynouard donne une quantité d'exemples des altérations du latin rustique. Il estime (p.50) que le nouveau marquage des cas grammaticaux ( $-s$ pour le nominatif singulier et l'accusatif pluriel vs. $-\phi$ pour le nominatif pluriel et l'accusatif singulier) a permis aux troubadours l'inversion poétiquement avantageuse des fonctions grammaticales. ${ }^{4}$ L'auteur passe en revue les nouveaux pronoms personnels, possessifs, démonstratifs, relatifs, indéfinis (p.51-65), puis la formation des verbes, modes, temps et voix (p.65-86) avec la conjugaison combinée en esser et estar et la formation du futur et du conditionnel ${ }^{5}$, il examine l'origine du que complétif (p.86) et des prépositions, adverbes et conjonctions (p.87-105). Quant à la section principale développant la grammaire de la langue romane, elle aborde les différentes parties du discours selon une disposition classique ${ }^{6}$.

\section{- $\quad$ SCHLEGEL (1818) et DIEZ (1820)}

Dans son double compte rendu de 1820, Diez dit des Observations sur la littérature provençale de A.W. Schlegel que «ce n'est pas seulement un compte rendu de l'ouvrage de Raynouard », mais il est vrai que leurs 82 pages du texte principal (sans intertitres) suivies de 41 pages de note en constituent essentiellement un commentaire critique ${ }^{7}$. Même si globalement Schlegel corrobore les observations de Raynouard, les points les plus intéressants sont ses marques occasionnelles de désaccord. C'est le cas p.23 à propos d'une déclaration de Raynouard sur le 'théotisque' - que Pierre de Cazeneuve, cité par Schlegel, appelle au début du $17^{\mathrm{e}}$ siècle le teudisque, variante de l'actuel tudesque, toutes ces variantes 
provenant de deutsch - et le gotique. Schlegel tenait en particulier à rappeler la qualité linguistique de la traduction de la Bible par l'évêque goth Ulfila. Je dispose les deux extraits en vis-à-vis :

\begin{tabular}{l|l}
\multicolumn{1}{c|}{ Raynouard cité par Schlegel, p.23 } & \multicolumn{1}{c}{ Schlegel, p.23 } \\
\hline Le mélange de ces peuples qui renonçoient à & (..) ces peuples guerriers et peu littéraires étoient \\
leur idiome grossier, et adoptoient l'idiome des & fort attachés à leur langue, quelle qu'elle fût, aux \\
vaincus, par la nécessité d'entretenir les & $\begin{array}{l}\text { souvenirs nationaux, aux chants hérö̈ques qu'elle } \\
\text { rapports religieux, civils et domestiques, ne } \\
\text { pouvoit qu'être funeste à la langue latine. La } \\
\text { décadence fut rapide. }\end{array}$ \\
$\begin{array}{l}\text { Clovis un chantre goth, qui savoit réciter les } \\
\text { antiques exploits de sa nation. }\end{array}$
\end{tabular}

Ce faisant, il précise que les Francs n'ont cessé de parler le théotisque « qu'après la séparation finale des empires de France et d'Allemagne, c'est-à-dire au commencement du dixième siècle » et il expose la vision dès lors classique de l'effet des migrations germaniques sur le latin populaire :

Les conquérants barbares (...) furent (...) forcés d'apprendre aussi le latin pour se faire entendre, mais ils le parloient en général fort incorrectement; surtout ils ne savoient pas manier ces inflexions savantes, sur lesquelles repose toute la construction latine. Les Romains, c'est-à-dire les habitans des provinces, à force d'entendre mal parler leur langue, en oublièrent à leur tour les règles, et imitèrent le jargon de leurs nouveaux maîtres (...) [les] désinences supprimées servoient à marquer d'une manière très-sensible la construction des phrases, et la liaison des idées ; il falloit donc y substituer une autre méthode, et c'est ce qui donna naissance à la grammaire analytique. (Schlegel 1818, p.24-25)

Schlegel est en accord avec Raynouard sur le cheminement analytique interrompu de ce que celui-ci appelle la «langue romane » et assimile au provençal des troubadours. Mais il refuse de voir dans cette langue «un intermédiaire entre le latin et les diverses langues modernes qui en dérivent» (p.39). Sa perplexité vient de ce que «l'italien et l'espagnol sont bien visiblement plus près du latin que le provençal » (p.43) et il poursuit (p.50) : «M. Raynouard a fort bien prouvé l'ancienneté des dialectes romans, mais non pas leur identité dans les diverses provinces », même s'il admet qu'au bas moyen-âge l'extension géographique du provençal a été largement supérieure à ce qu'elle est devenue au $19^{\mathrm{e}}$ siècle. Dans le fragment qui suit, on notera qu'en ce début du $19^{\mathrm{e}}$ siècle, Schlegel qualifie le provençal de 'patois'. C'est certainement la renommée de l'œuvre de Raynouard qui a permis la redécouverte des Cours d'amour avec la fondation du Félibrige en 1854, de l'Armana Prouvençau un an plus tard et la publication de Mirèio de Frédéric Mistral en 1859, œuvre qui allait lui valoir le Nobel de littérature en 1904.

Depuis que ce dialecte a été condamné à n'être plus qu'un patois, les langues dominantes qui l'environnent, le français, l'italien et l'espagnol ont dû constamment gagner du terrain, soit en remplaçant l'ancien langage du pays, soit en l'altérant. (Schlegel 1818, p.51)

Cependant le provençal n'a pas été déprécié seulement par le poids sociolinguistique de ses grands voisins, il l'a été aussi par ses propres faiblesses, en particulier sa morphologie hybride mi-synthétique comme le latin, mi-analytique comme le français, l'italien ou l'espagnol, un « défaut des langues dont la formation n'est pas encore achevée »(p.57). Une manifestation de cet inachèvement, néfaste pour la grammaire, mais faste pour la versification, est selon Schlegel la variation extrême des formes des pronoms personnels et possessifs, l'abondance des particules et des paradigmes de conjugaison entachés de nombreuses irrégularités.

De son côté, Diez avance, dans le prolongement de Schlegel, des arguments intéressants à l'encontre de la thèse de Raynouard qui voulait que le 'théodisque' se soit perdu immédiatement après la mort de Charlemagne, peut-être même plus tôt :

Ses preuves ne se fondent en aucun cas sur une vision correcte de la vie interne des peuples et de leurs échanges, mais le plus souvent sur de simples mots isolés que l'histoire nous a transmis sans garantie de fiabilité. Comment devrions-nous nous convaincre qu'une seule et même langue ait pu s'imposer dans des pays qui ont été soumis à tant d'influences après la migration des peuples [germains] et que ne rassemblait aucun lien ni politique ni littéraire, sans vouloir par ailleurs nier que le processus évolutif de toutes ces langues méridionales ait présenté de grandes similitudes, plus encore dans les temps les plus anciens, avant 
que certaines aient commencé à prendre un caractère particulier, tandis que le provençal, l'organe de poètes exceptionnels dès cette époque était déjà aussi développé ? (Diez 1820, p.44, ma trad.)

Il se range cependant à la désignation, prônée par Raynouard, du provençal comme «langue romane », tout en s'accordant avec Schlegel sur la disparition tardive du théodisque ${ }^{8}$.

\section{- RAYNOUARD 1821 et DIEZ 1825, 1829}

Dans sa Grammaire comparée des langues de l'Europe latine dans leurs rapports avec la langue des troubadours, Raynouard complète ses observations de 1816 en comparant successivement les traits grammaticaux majeurs du provençal avec ceux du français (pp.xvi-xxviii), de l'espagnol (p.xxix), des patois d'Espagne (p.xxxvi), du catalan (p.xxxviii), du portugais (p.xl), de l'italien (p.xlvii) et du valaque ou moldave (p.lxi). Dans la section consacrée à la comparaison entre le roman et le français, il examine en particulier l'évolution des voyelles, que le tableau 2 cherche à résumer avec un exemple de chaque type de formation.

\begin{tabular}{|l|l|l|l|}
\hline \multicolumn{2}{|c|}{ roman } & \multicolumn{2}{c|}{ français } \\
\hline al & corporal & el & corporel \\
-at & libertat & -et & libertet \\
el & (bel) & eau & (beau) \\
l & almone & u & aumone \\
au & auratge & o & orage \\
o & boca & ou & boche $>$ bouche \\
o & foc, loc & uec $>$ eu & fuec, luec $>$ feu, lieu \\
\hline
\end{tabular}

\begin{tabular}{|l|l|l|l|}
\hline \multicolumn{2}{|c|}{ roman } & \multicolumn{2}{c|}{ français } \\
\hline or & razor & our/eur/oir & rasoir $(+\mathrm{p} .59)$ \\
os & & eux & p.122sq \\
-u, -v & votiu & -f & votif \\
e & mel & ie & miel \\
a & fam & ai & faim \\
en & fren & ein & frein \\
e & rei, borges & o, oi & roi, bourgeois \\
\hline
\end{tabular}

Tableau 2 : L'évolution des voyelles du roman en français selon Raynouard (1821, pp.xvi-xxiv)

Son raisonnement tient à nouveau en deux points : [1] la langue romane entretient des rapports distincts avec chacune des langues qui en sont issues ${ }^{9}$, et [2] une reconstruction (il n'emploie pas encore cette terminologie) des origines de la phonologie et de la morphologie de chacune des langues romanes conduirait à une langue primitive commune ${ }^{10}$. Ainsi, fort de sa connaissance exceptionnelle du corpus des troubadours, Raynouard reconstitue une version romane d'un passage de la Divine comédie (p.385) et deux strophes d'une chanson en français de Raoul de Beauvais (p.387) pour asseoir sa thèse du « roman commun ». Quant aux raisons de la disparition du provençal come langue littéraire, il les voit dans les actes du gouvernement français rédigés en français et l'attrait de la cour parisienne dès le $13^{\mathrm{e}}$ siècle :

ainsi la langue romane, ce bel idiome des troubadours, n'avait plus aucun moyen de se produire et de se maintenir hors du cercle étroit de l'usage domestique dans lequel elle resta reléguée. (Raynouard 1821, p.390)

Raynouard se livre à un exercice de pensée consistant à imaginer ce qui aurait résulté d'un gouvernement de la France à partir de l'espace occitan (p.391). La langue des troubadours, « devenant la langue de la France entière, aurait offert beaucoup d'avantages dont la plupart n'existaient déjà plus ou cessèrent bientôt d'exister dans la langue des trouvères », entre autres l'opposition double, déjà mise en avant en 1816 (cf. plus haut), entre les désinences $-\varnothing$ et -s pour le marquage du nominatif et de l'accusatif.

Dans les années qui suivent, Diez fait paraître deux ouvrages $(1825,1829)$ destinés à faire connaître l'œuvre poétique des troubadours par des traductions en allemand, mais il n'y adjoint pas d'annexe linguistique, soit parce qu'il était convaincu que Raynouard avait tout dit, soit plutôt parce qu'il se réservait pour sa grande Grammaire des langues romanes en gestation.

\section{Le provençal à l'époque des œuvres de la maturité de Raynouard et Diez (1836-1838)}

Dans l'introduction à l'Essai sur les cours d'amour de Diez (all. 1829, fr.1842), son traducteur Ferdinand de Roisin rend hommage à la contribution des romanistes allemands à l'entreprise des philologues de l'École des Chartes où le provençal joue un rôle central : 
(...) les Francisque Michel, les Paulin Paris, les Jabinal, en un mot toute une ardente milice formée à l'École des Chartes, s'adonnent avec ferveur à cette entreprise de patriotisme littéraire; l'Allemagne, de son côté, ne cesse de seconder leurs efforts, soit en restituant à la France ces productions de la muse romane égarées sur le sol étranger, soit en élaborant, avec cette consciencieuse industrie en matière de recherches qui la distingue, des ouvrages didactiques; soit enfin en soumettant au creuset d'une saine critique les nombreux produits de la presse française. (Roisin 1842, p. XX)

Entre les lignes, on comprend que les philologues français doivent rester les maîtres d'œuvre, et cela va de soi puisqu'il s'agit d'une «entreprise de patriotisme littéraire », et aussi longtemps que Raynouard est aux commandes du haut de sa chaire au Collège de France et de ses deux fauteuils d'académicien ${ }^{11}$, que A.W. Schlegel se contente de recenser (et d'encenser) le $1^{\mathrm{er}}$ volume linguistique du Choix des poésies originales des troubadours de Raynouard et que Diez se concentre sur l'édition critique de la poésie des troubadours (cf. 1825, 1829), l'ambition des philologues patriotes n'est pas concurrencée. Il n'en est cependant plus de même quelques années plus tard. Certes Raynouard publie encore en 1836 un complément de 92 pages à sa grammaire comparée de 1821, sous le titre Influence de la langue romane rustique sur les langues de l'Europe latine, suivi en $1838 \mathrm{du} 1^{\mathrm{er}}$ volume de son Lexique roman ${ }^{12}$ mais au même moment Diez fait paraître successivement les trois tomes de sa Grammatik der romanischen Sprachen, qui vont réellement fonder la linguistique romane.

\section{- La langue romane rustique et le lexique roman (Raynouard 1836, 1838)}

L'opuscule de 1836 (Influence de la langue romane rustique sur les langues de l'Europe latine) développe les observations de 1821 autour du commentaire minutieux du texte des deux serments de Strasbourg (842), celui de Louis le Germanique et celui « du peuple français ». Raynouard observe en particulier (p.5) que dans le premier serment figure salvament au lieu de salvatio (et dans le serment du peuple français : sagrament) et il ajoute :

Ce qui est plus étonnant c'est que le substantif salvament se retrouve dans les six langues néolatines :

TROUB. Salvament. CAT.Salvament. ESP.Salvamiento.

PORT. Salvamento. IT. salvamento. FR. Saulvement.

De même on trouve adjudha dans les deux serments au lieu de adjutorium < adjutor < adjuvo, cf. fr. aide. Et cette formation se retrouve aussi dans les six langues néolatines, ce qui permet à Raynouard de conclure par une question rhétorique (p.8) :

Cette décomposition de la langue latine et la recomposition romane ne démontrent-elles pas, jusqu'à la dernière évidence, que cette langue rustique, dont il nous reste ces deux fragments de l'an 842, possédait à un haut degré l'art de créer, avec les éléments latins, les mots qui lui convenaient pour exprimer ou plus clairement ou plus rapidement les sentiments et les idées ? (Raynouard 1836, p.8)

L'essentiel de l'opuscule (pp.28-83) est constitué de vingt mini-glossaires portant sur six langues divisées en deux tableaux, par ex. pour le champ conceptuel de la nourriture, des aliments et des boissons (§II p.28sq) :

\begin{tabular}{|c|c|c|c|c|c|}
\hline Trout & Cat. & tsp. & 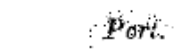 & it: & Fr \\
\hline Ytonlle. & Titualla. & Yitidilla & Vitualia: & Tituagitia & Victuatle: \\
\hline Biscoit. & Bestuyt & Bupadio & Biseauto. & Biscotto & Biscinte \\
\hline Pebrada & Pebrada & Pebrada & Poniada, & Pevereda. & Foivrade. \\
\hline $\begin{array}{l}\text { Cancla } \\
\text { Gara dxyo }\end{array}$ & $\begin{array}{l}\text { Caneyla } \\
\text { Gisat }\end{array}$ & $\begin{array}{l}\text { Ganeta } \\
\text { Oisna }\end{array}$ & Gapela & Cannela & Cannelle. \\
\hline Salran. & Safré & Azafran. & Glara, & Chiara & Glare (dould \\
\hline Claret & Glate & Glareie: & $\begin{array}{l}\text { Acafráo } \\
\text { Clarete }\end{array}$ & $\begin{array}{l}\text { Quferato: } \\
\text { Glaretio. }\end{array}$ & Cafrán. \\
\hline
\end{tabular}

et il est suivi de sept tableaux comparatifs complémentaires (p.84-92). 
De son côté, le Lexique roman bénéficie d'une publication posthume (Raynouard est mort en 1836) en six tomes de 1838 à 1844. Les tomes 2-5 constituent le lexique proprement dit (A-C ; D-K ; L-P ; Q-Z). Ils sont précédés d'un tome 1 d'introduction et suivis d'un tome 6 de mise à jour.

Le tome 1 se subdivise en un préambule linguistique (p.v-lxxxviii) et une anthologie de poésies des troubadours (p.1-580). Raynouard expose en ces termes le contenu du préambule :

Je ferai précéder ce nouveau choix de poésies :

$1^{\circ}$ De quelques observations historiques sur la langue rustique romane, type primitif, centre commun des six langues de l'Europe latine.

$2^{\circ}$ Après avoir rappelé les faits historiques qui prouvent la filiation des langues néolatines, j'offrirai quelques observations sur l'étude philosophique des langues.

$3^{\circ}$ Ce travail sera suivi de l'exposé des motifs qui doivent porter les littérateurs à étudier la langue des troubadours, afin de mieux connaître et de mieux apprécier les autres idiomes néo-latins.

$4^{\circ}$ Enfin, je donnerai un résumé des règles grammaticales de la langue des troubadours, de la romane provençale, en y ajoutant quelques observations nouvelles sur divers points grammaticaux, pour préparer à la lecture et à l'intelligence des nouveaux documents poétiques que je publie.

Le tome 1 est donc autosuffisant, cependant les tomes suivants fournissent - dans le prolongement de l'opuscule de 1836 - des données précieuses sur le destin des mots du latin 'rustique' en roman 'commun' et dans chacune des langues 'néo-latines'.

\section{- La place du provençal dans la Grammaire des langues romanes de Diez (1836-1838)}

Si le provençal joue le rôle d'une plaque tournante dans la vision de Raynouard, il n'en est pas de même dans la Grammatik der romanischen Sprachen de Diez, en particulier dans le tome 2 consacré à la morphologie grammaticale et lexicale ${ }^{13}$. Dans chacune des sections consacrées aux paradigmes de déclinaisons et de conjugaisons des diverses langues romanes, Diez commence par des considérations générales, avant d'aborder chaque langue isolément. Le provençal y figure régulièrement entre le portugais et le français. Ses déclinaisons substantivales figurent ${ }^{14}$ dans la première édition allemande p.31-35 et dans l'édition française de G. Paris et A. Brachet (1874) p.31-40, les déclinaison adjectivales dans l'éd. all. p.55-57, dans l'éd.fr. p.64-67, les déclinaisons pronominales dans l'éd. all. p.80-84, dans l'éd. fr. p.88-95 et les conjugaisons dans l'éd. all. p.166-183 et dans l'éd. fr. p.180-204. Diez accorde donc au provençal une importance sans commune mesure avec ce à quoi il a été réduit au $19^{\mathrm{e}}$ siècle (et dans l'édition française on trouve des paragraphes sur le provençal moderne), mais sans le présenter, contrairement à Raynouard, comme un intermédiaire irréfutable entre le latin 'rustique' et les langues 'néo-latines'.

\section{La montée en puissance de la romanistique allemande ${ }^{15}$ et la place du provençal (1863-1868)}

Nous disposons d'un précieux témoignage sur l'impact croissant des romanistes allemands dans la seconde moitié du siècle, et sur l'attention qu'ils portent au provençal, avec l'article de Gaston Paris, $L a$ philologie romane en Allemagne, publié en 1864, qui complète sa préface d'une plaquette consacrée un an plus tôt à la traduction de l'Introduction du premier volume de la Grammatik de Diez ${ }^{16}$.

En 1863, K.A.F. Mahn fait à Meissen (en Saxe) une conférence mémorable sur la naissance, la signification et les objectifs de la philologie romane que G. Paris entreprend de commenter dans son article. Mahn regrette que trop peu d'universités allemandes et suisses et d'académies s'intéressent à la philologie romane et anglaise (p.436), mais Paris constate amèrement qu' «en France, il n'y a pas une seule Faculté des Lettres où on fasse des cours ayant trait à la philologie romane » ${ }^{17}$ et il a l'humour d'ajouter à propos du français (p.437) :

Mais ne pourrions-nous pas sauver au moins les apparences, avoir l'air de nous intéresser quelque peu à cette langue dont nous sommes si fiers? » [italiques de l'auteur]

Mahn, fort de l'impulsion donnée par la Grammatik de Diez, explique à ses collègues comment diffuser la philologie romane à travers le réseau universitaire allemand, et Paris constate effectivement que les universités de Leipzig et de Vienne ont ouvert des chaires de philologie romane respectivement en 1862 
pour Adolf Ebert et en 1860 pour Adolfo Moussafia (spécialement pour l'italien, cf. François à paraître, $\S 1.3 .2 .2)$. Concernant spécifiquement l'ancien provençal, Mahn déclare qu'on ne peut pas assez en recommander l'étude et l'enseignement et il lui attribue, dans l'étude des langues romanes, « le rôle du gothique dans celle des langues germaniques » (Paris 1963, p.440). Apprenant qu'une société savante de Berlin a dégagé un financement conséquent pour permettre à un philologue allemand de parcourir toute l'Italie à la recherche de manuscrits de la langue des troubadours à éditer, il fait mine de s'étonner :

Ici encore il est diffcile de ne pas trouver assez étrange qu'une société berlinoise fasse pour nos troubadours ce que ne font ni notre gouvernement ni nos Académies (...) on n'aime pas à voir des étrangers s'occuper des cendres de vos pères et leur procurer des monuments dignes d'elles : seulement il arrive quelquefois qu'on s'avise trop tard de leur disputer cet honneur (p.441-2)

ce qui ne l'empêche pas de conclure avec gratitude (p.445) :

Il est heureux, en attendant, que l'Allemagne fasse notre besogne; son zèle entretient le feu sacré et encourage chez nous les quelques obstinés qui s'adonnent encore à une science que n'encouragent ni les faveurs officielles ni l'attention publique.

\section{Le provençal à l'époque de la suprématie de la romanistique allemande (1885-1909)}

Au tournant du $20^{\mathrm{e}}$ siècle, le provençal ne semble plus jouir d'une attention particulière, ni en France où plus aucune publication majeure n'est consacrée à sa grammaire ou à son lexique, ni même en Allemagne où l'index de l'Introduction à l'étude de la linguistique romane [Einführung in das Studium der romanischen Sprachwissenschaft] de Meyer-Lübke (1909) mentionne beaucoup moins de mots provençaux (195) que de mots français (535) ou italiens (471).

Cependant après l'imposant glossaire de la Crestomathie provençale de Bartsch en 1868 (environ 6000 mots) et la succession des grammaires du provençal par Raynouard (1816, 1821, 1838), Diez (18361838) et Bartsch, on peut simplement en conclure que les chercheurs disposent désormais d'un attirail philologique satisfaisant. K.A.F. Mahn consacre un ouvrage en 1885 à la phonétique du provençal [Lautlehre der altprovençalischen Sprache], sans doute aiguillonné par la nouvelle «physiologie des sons » d'Eduard SIEVERS (1876), tandis qu'Emil Levy publie successivement deux dictionnaires, le premier en allemand en 1894, présenté comme un supplément au Lexique roman de Raynouard (1838), y apportant corrections et compléments [Provençalisches Supplement-Wörterbuch - Berichtigungen und Ergänzungen zu Raynouards Lexique Roman] et le second en français sous le titre Petit dictionnaire provençal-français (1909).

Mais lorsque paraît en 1905 la seconde édition profondément remaniée du premier tome linguistique du Traité de philologie romane [Grundriß der romanischen Philologie] dirigé par Gustav Gröber, il n'est plus question de réserver un chapitre particulier à la présentation de la langue provençale. Le maître d'oeuvre préfère demander à Hermann SUCHIER (1848-1914) de rédiger un seul gros chapitre (p.712841) consacré aux deux langues française et provençale et à leurs dialectes [Die französische und provenzalische Sprache und ihre Mundarten]. Ainsi, l'époque de l'étude systématique de la grammaire et du lexique du provençal est bien terminée.

\section{6. Épilogue : Le point aveugle du roumain}

On aura noté que les six langues que compare Raynouard $(1821,1836)$ sont d'une part la langue des troubadours, d'autre part le catalan, l'espagnol castillan, le portugais, l'italien et le français, la première étant considérée, en tant que 'langue romane', comme le goulot d'étranglement par lequel a dû passer le 'latin rustique' pour donner naissance à l'ensemble de l'espace linguistique roman. Mais le roumain - que Diez appelait le 'valaque-moldave' - brille par son absence, et c'est bien compréhensible : par quelle extraordinaire migration aurait-il pu naître du provençal, alors qu'on sait qu'il a été la langue des colons romains en Dacie après la conquête de l'empereur Trajan ?

Dès lors que le roumain est pris en compte, la thèse de Raynouard est pour le moins amputée de l'espace que Christian Lehmann (université d'Erfurt, en ligne) appelle 'Balkanromanisch' ou 'Ostrandromanisch' (balkanoroman / roman de la marge orientale). Ce typologue et également spécialiste du latin distingue 
trois espaces romans de premier ordre, deux espaces occidental et oriental indépendants et un espace central recouvrant partiellement les deux autres. Cette disposition donne lieu à quatre espaces de second ordre : roman de la marge occidentale (ibéroroman), roman central-occidental (galloroman), roman central-oriental (italoroman) et roman de la marge orientale (balkanoroman). Et Lehmann, en typologue des langues et théoricien majeur de la grammaticalisation, fait une observation décisive : les deux variantes marginales, occidentale (l'ibéroroman) et orientale (le balkanoroman) présentent des caractères communs qui ne peuvent pas avoir été des innovations communes, mais qui résultent d'archaïsmes communs conservés en l'absence des innovations qui caractérisent le roman central (galloroman et italoroman $)^{18}$. On aurait aimé que Lehmann détaille ces archaïsmes communs, car on se souvient que Schlegel (cf.§2) voyait dans les défauts du provençal, en particulier son caractère mi-synthétique (par son ascendance latine), mi-analytique (par sa descendance romane), la caractéristique d'une évolution inachevée. C'est sans doute sur ce seul plan de la transition entre deux morphosyntaxes, synthétique puis analytique, que l'ancien provençal a représenté un maillon décisif pour les langues romanes occidentales.

\section{Références bibliographiques ${ }^{19}$}

BARTSCH K. (1854), Provenzalisches Lesebuch, mit einer literarischen Einleitung und einem Wörterbuche. (non consulté, rééd. Genève : Slatkine 1974).

BARTSCH K. (1868) ${ }^{\mathrm{A}}$, Chrestomathie provençale. (Édition Gallica, Berlin 1892)

DIEFENBACH Lorenz (1832) ${ }^{\mathrm{A}}$, Über die jetzigen romanischen Schriftsprachen. Leipzig : Ricker (actuellement disparu du catalogue de Archive.org).

DiEz F. (1825), Über die Minnehöfe, Beiträge zur Kenntnis der romanischen Poesie. Berlin; trad.fr. (Essai sur les cours d'amour) ${ }^{\mathrm{G}}$ par F. de Roisin, Lille 1842.

DiEz F. (1829) ${ }^{\mathrm{A}}$, Leben und Werke der Troubadours. (Édition Archive.org $\bullet$ Leipzig : Ambrosius Barth 1882).

DIEZ F. (1836-1838) ${ }^{\mathrm{A}}$, Grammatik der romanischen Sprachen. (Édition Archive.org $\bullet$ Bonn : Eduard Weber 1844)

DiEZ F. $(1863)^{\mathrm{G}}$, Introduction à la grammaire des langues romanes. Traduit par G. Paris. Paris / Leipzig : Franck

Diez F. (1874-1876) ${ }^{\mathrm{G}}$, Grammaire des langues romanes. Traduit par G. Paris, A. Brachet et Alfred Morel-Fatio. Paris : Franck

DiEZ F. $(1883)^{\mathrm{A}}$, Kleinere Arbeiten und Rezensionen [Travaux mineurs et comptes rendus] édition posthume par Hermann Breymann, Munich : Oldenburg (contient un compte rendu 1920 sur Raynouard 1816 et Schlegel 1818, pp.39-48).

FRANÇOIS J. (2014), La mise en place de la philologie et de la linguistique romanes dans l'Allemagne du $19^{\mathrm{e}}$ siècle. Romanistisches Jahrbuch 64 (2013) : 33-60.

FrANÇOIS J. (à paraître), Le siècle d'or de la linguistique en Allemagne : de Humboldt à Meyer-Lübke. Limoges : Lambert-Lucas.

La Curne de Saint-Palaye J.B. (1774) ${ }^{\mathrm{G}}$, Histoire littéraire des troubadours. Édition posthume par l'abbé Millot. (Édition Gallica $\triangleright$ Genève : Slatkine 1967)

LEHMANN Ch. (en ligne), Genetische Verwandtschaft unter Sprachen $\triangleright$ http://www.christianlehmann .eu/ling/wandel/genet_verwandtschaft.php

LEVy E. (1894) ${ }^{\mathrm{A}}$, Provenzalisches Supplement-Wörterbuch - Berichtigungen und Ergänzungen zu Raynouards Lexique Roman [Supplément au dictionnaire provençal - corrections et compléments au Lexique roman de Raynouard] (Édition Archive.org Leipzig : Reisland 1904)

LÉvy E. (1909), Petit dictionnaire provençal-français (Réédition \ Culture provençale et méridionale, 1991)

MaHn K.A.F. (1863), „Ueber die Entstehung, Bedeutung, Zwecke und Ziele der romanischen Philologie“ [Sur la naissance, la signification et les objectifs de la philologie romane]. Berlin (cité par Paris 1863)

MAHN K.A.F. (1885), Lautlehre der altprovençalischen Sprache. Köthen (non consulté)

MEYER-LÜBKE W. (1909) ${ }^{\text {A }}$, Einführung in das Studium der romanischen Sprachwissenschaft. Heidelberg : Winter.

Mistral F. (1861) ${ }^{\mathrm{G}}$, Mirèio. Paris : Charpentier

PARIS G. (1864) ${ }^{\mathrm{P}}$, “La philologie romane en Allemagne”. Bibliothèque de l'École des Chartes, volume 25 : 435-445.

RAYNouARD F.J.M. $(1816)^{\mathrm{G}}$, Choix des poésies originales des troubadours. Paris : Didot.

RAYNOUARD F.J.M. $(1821)^{\mathrm{G}}$, Grammaire comparée des langues de l'Europe latine, dans leurs rapports avec la langue des troubadours Paris : Didot. 
RAYNOUARD F.J.M. $(1836)^{\mathrm{G}}$, Influence de la langue romane rustique sur les langues de l'Europe latine Paris : Crapelet.

RAYNOUARD F.J.M. $(1838)^{\mathrm{G}}$, Lexique roman ou Dictionnaire de la langue des troubadours comparée avec les autres langues de l'Europe. Paris: Silvestre.

RochEGUdE H.P.de (1819) ${ }^{\mathrm{Go}}$, Essai d'un glossaire occitanien: pour servir à l'intelligence des poésies des troubadours. Toulouse : Benichou.

SCHLEGEl A.W. $(1818)^{\mathrm{G}}$, Observations sur la langue et la littérature provençales. Paris : Librairie Latine-GrecqueAllemande.

Suchier H. (1904) ${ }^{\mathrm{A}}$, "Die französische und die provenzalische Sprache und ihre Mundarten”. In : G. Gröber (éd.), Grundriß der romanischen Philologie, p.712-840. Strasbourg : Trübner.

${ }^{1}$ Paulin Paris, lui-même médiéviste, avait envoyé son fils Gaston poursuivre ses études philologiques en Allemagne, où il fit la connaissance de Diez dont il devint un disciple fervent.

${ }^{2}$ Le premier volume de 1816 fournit les bases linguistiques de l'étude des poésies des troubadours. Les volumes littéraires se sont succédé en 1817 (vol.2), 1818 (vol.3), 1819 (vol.4) et 1820 (vol.5). La Grammaire comparée de 1821 constitue le vol.6 de la collection.

${ }^{3}$ Édition en trois volumes (plus de 1400 pages) due à l'Abbé Millot, reprise par l'éditeur Slatkine à Genève en 1967.

${ }^{4}$ Cf. p.50 : Peut-on assez admirer cette industrie grammaticale, qui n'a existé dans aucune autre langue, industrie qui ensuite permit et facilita aux troubadours la grâce et la multitude des inversions à-la-fois les plus hardies et les plus claires? Les anciens monuments de la langue romane offrent l'heureux emploi de ce signe caractéristique.

${ }^{5}$ La description de ce mode de formation en deux temps, analytique par auxiliation, puis synthétique par suffixation des restes de l'auxiliaire, a été reprise par L. Diefenbach en 1832 dans son traité pionnier pour l'école allemande auquel Diez a rendu hommage.

${ }^{6}$ Chap.I : Articles (p.110) ; chap.II : Substantifs (p.121) ; chap.III : Adjectifs (p.138) ; chap.IV Pronoms (p.155); chap.V : Noms de nombres (p.257); chap.VI : Verbes (p.263); chap.VII : Adverbes, prépositions, conjonctions (p.343) ; chap.VIII : Locutions particulières, idiotismes de la langue romane (p.432)

${ }^{7}$ En dehors des pp.14-17 consacrées à une présentation détaillée du classement morphologique des langues de Humboldt avant que son auteur lui-même le fasse connaître à partir de 1820. Diez (1820, p.48) indique que Schlegel concevait ses Observations comme l'introduction à un Essai historique sur la formation de la langue française en plusieurs volumes et il ajoute qu'il attend ardemment la poursuite de ce projet. Cependant A.W.Schlegel, tout comme son frère Friedrich, avait plusieurs cordes à son arc comme philosophe, théoricien de l'art et en 1819 premier professeur de sanskrit (à l'université de Bonn), et ce projet éditorial ne vit finalement pas le jour.

${ }^{8}$ Diez cite à cette fin le jugement de Pierre-Nicolas Bonamy : Les classes inférieures des francs s'étaient peut-être habituées depuis quelques siècles à la langue romane et ont progressivement abandonné leur propre langue, tandis que les classes supérieures n'ont pu s'y décider qu'après le démantèlement [de l'empire carolingien en 842], pour attester ainsi la fondation d'un royaume distinct. (Mémoire de l'Académie des Inscriptions et Belles-Lettres, 1756, t.XXIV)

${ }^{9}$ cf. p.376 : les passages que je citerai, quoique identiquement romans et français, romans et espagnols, romans et portugais, romans et italiens, ne conservent ce rapport d'identité qu'entre le roman et chaque langue isolée ; c'està-dire que le passage qui est à-la-fois roman et français n'est plus français et espagnol, et de même le passage qui est roman et espagnol n'est plus espagnol et français ou italien, etc.

${ }^{10}$ De même qu'on a parlé d'un «germanique commun» antérieur au gotique, au nordique et au vestique. Cf. Raunouard 1821, p.384 : Il est aisé de reconnaître les principales modifications qui constituent les diverses nuances par lesquelles les autres langues de l'Europe latine diffèrent de la romane primitive; et si l'on se prêtait à ce que ces modifications disparussent dans chacune des langues, elles redeviendraient identiques avec celle des troubadours.

${ }^{11}$ Raynouard a été élu en 1816 à l'Académie des Inscriptions et Belles Lettres et dès 1807 à l'Académie Française (secrétaire perpétuel en 1817).

${ }^{12}$ Le sous-titre en est particulièrement développé : Dictionnaire de la langue des troubadours comparée avec les autres langues de l'Europe latine, précédé de nouvelles recherches historiques et philologiques, d'un résumé de la grammaire romane, d'un nouveau choix des poésies originales des troubadours, et d'extraits de poëmes divers.

${ }^{13} \mathrm{cf}$. https://archive.org/stream/grammatikderrom00unkngoog\#page/n5/mode/2up. 
${ }^{14}$ Le contenu des sections de l'édition allemande originale de 1838 et de l'édition française de 1874 diffère légèrement, car G. Paris et $\mathrm{A}$. Brachet ont traduit une édition postérieure corrigée et complétée, avec en particulier la présentation des variantes du provençal moderne et du catalan en queue de section sur le provençal.

${ }^{15}$ Cf.François (2014) pour une présentation générale de la genèse et de l'évolution de la romanistique en Allemagne.

${ }^{16}$ Je ne reviens pas sur l'importance pour le provençal de l'œuvre, à cette époque, de Karl Bartsch, évoquée dans la section 1.

${ }^{17}$ Les deux seuls lieux où s'enseigne la philologie romane à l'époque sont l'École des Chartes avec Francis Guessard, puis Paul Meyer, et le Collège de France avec Raynouard, puis Paris et le même Meyer, spécialiste entre autres du provençal.

18 „Die Pointe ist, daß Randromanisch Gemeinsamkeiten aufweist, die nicht DURCH GEMEINSAME NEUERUNGEN entstanden sind (was angesichts geographischer Isolierung auch schwerlich möglich wäre). Vielmehr rühren sie daher, daß die Randgebiete die Neuerungen des Zentralromanischen nicht mitgemacht, also GEMEINSAME ARCHAISMEN bewahrt haben“" [http://www.christianlehmann.eu/ling/wandel/genet_verwandtschaft.php].

${ }^{19}$ Les ouvrages et articles consultables sont indiqués par un exposant : base ARCHIVE.ORG $>$ A. ; base GALLICA $\checkmark$ G, base Google.books $>$ Go ; portail Persée : P. 